\title{
The review of COVID-19 and the current progress in intervention and diagnostics
}

\author{
Chunyun Chen ${ }^{1, \dagger}$, Xi Chen ${ }^{2, \dagger}$, Liwei Guo ${ }^{3, \dagger, *}$ and Kanghui Zou ${ }^{4, \dagger}$ \\ ${ }^{1}$ Xinxiang Medical University, China \\ ${ }^{2}$ Shanghai Pinghe School, China \\ ${ }^{3}$ Changchun University of Science and Technology, School of Life Science and Technology, China \\ ${ }^{4}$ Nanjing Jinling Highschool, International Department, China \\ These authors contributed equally.
}

\begin{abstract}
COVID-19 was first reported in Wuhan city of Hubei Province of China in December 2019, becoming a pandemic declared by the world health organization. This article is a review of the novel coronavirus disease (COVID-19). It typically informs the genome structure of the SARS-CoV-2 and its pathogenic mechanisms, concludes a series of non-pharmaceutical control methods, and focuses on several testing measures. The inventions of the disease treatments remain an important challenge to all medical institutions while a series of medications have been brought to the public.
\end{abstract}

\section{Introduction}

At the end of 2019, a new type of coronavirus called SARS-CoV-2 appeared, causing an outbreak of viral pneumonia worldwide. Infectious pneumonia caused by SARS-CoV-2 infection is also known as Coronavirus disease 2019 (COVID-19), which is highly pathogenic and contagious, and spread quickly to all parts of the world [1]. In this Review, we describe the basic virology of COVID-19, including genomic characteristics and receptor use, highlighting its key difference from previously known coronaviruses [2]. Various prevention and control measures such as non-drug intervention could cut off its pathological transmission. This article combines the literature to summarize and analyze the pathogenic mechanism, prevention, and control status, diagnosis, and treatment of SARS-CoV-2.

\section{Novel coronavirus}

\subsection{Genome structure and related protein characteristics}

The genome of SARS-CoV-2 contains 29,903 bases and encodes approximately 9,860 amino acids. The similarity with SARS-CoV is about $80 \%$, and the similarity with MERS-CoV is about $50 \%$ [3]. The genome structure of COVID-19 contains two flanking untranslated regions (UTRs), a 5'open reading frame (ORF1a/b), and several open reading frames encoding structural proteins $[4,5]$. ORF1a and ORF1b mainly encode nonstructural proteins (NSPs) related to viral replication. The open reading frame at the 3 'end mainly encodes viral structural proteins: spike protein(S), an envelope protein(E), membrane protein $(\mathrm{M})$, and nucleocapsid protein( $\mathrm{N})[6,7]$. The $\mathrm{S}$ protein is a multifunctional molecular machine that mediates coronavirus entry into host cells. S protein is a class of homotrimeric fusion glycoprotein containing two functional domains (S1 and S2) [8].

\subsection{Pathogenic mechanism}

The invasion of COVID-19 is mainly through the receptor-binding domain on the virus and the related receptors on the target cell. The main target receptor recognized by COVID-19 is similar to SARS-CoV, using angiotensin-converting enzyme 2 (ACE2) on the cell surface as the corresponding recognition receptor [9]. When the S protein of COVID-19 interacts with the ACE2 protein on the cell surface, the $\mathrm{S}$ protein is cleaved into two subdomains, S1 and S2. The fusion peptide (FP) domain of the S2 subunit is embedded in the host cell membrane, while the transmembrane (TM) domain is embedded in the virus shell. Subsequently, FP and TM together form a hexamer hairpin structure, which shortens the spatial distance between the virus and the host cell, making the membrane fusion more conducive to the virus entering the cell [10]. A recent study compared the binding affinity of the S protein in SARS-CoV and SARSCoV-2 to the receptor ACE2 and found that the binding affinity of SARS-CoV-2 and ACE2 is 10-20 of SARS$\mathrm{CoV}$ Times. This may be the reason for the high pathogenicity and widespread of COVID-19 [11].

After the membrane fusion between the virus and the cell, the viral genome is released into the host cell's cytoplasm. Among them, ORF1a and ORF1b are translated into viral replication-related replication 
complex proteins, which are further cleaved into 16 independent non-structural proteins under the host's action and viral proteases [4]. These non-structural proteins include RNA-dependent RNA polymerase, which participates in the replication of the viral genome. The full-length positive-strand genomic RNA is first transcribed into a negative-strand template, and new viral genomic RNA is synthesized from the template. After RNA replication is complete, the structural proteins $\mathrm{N}, \mathrm{S}$, $\mathrm{E}$, and $\mathrm{M}$ are also translated and synthesized. The S, E, and $M$ proteins are embedded in the endoplasmic reticulum. They are transported to the endoplasmic reticulum-Golgi intermediate compartment to assemble the $\mathrm{N}$ protein and the viral genome into mature virus particles. Finally, the virus particles are transported to the cell surface and released outside the cell through exocytosis, forming a new progeny virus to be released into the host [7].

\subsection{Pathological features}

The incubation period of COVID-19 is generally 5 to 6 days, and the average incubation period is 5.2 days. Once symptoms of infection appear, the viremia caused by virus replication reaches its peak within 2 to 5 days [12]. About $80 \%$ of infected people with COVID-19 is a mild disease, mainly manifested as respiratory and bronchial infections. These individuals usually show moderate symptoms similar to ordinary flu, including fever and dry cough, and disappear on their own within 6 to 10 days. However, in nearly $20 \%$ of patients, the virus infection progresses to the lower end of the trachea and even the lungs. The increase of pro-inflammatory cytokines in the lung recruits many white blood cells, further expanding the local inflammatory response [13]. The latest histological studies have shown that the central location of the lungs is the most susceptible part. Symptoms such as lung cell injury, diffuse alveolar injury, edema, and alveolar hemorrhage are accompanied by infection. In the pulmonary inflammation stage, the disease can rapidly progress to a severe infection characterized by acute respiratory distress syndrome (ARDS), a highinflammatory state with multiple organ dysfunction. In the most severe cases, the fatality rate is close to $10 \%$ [14].

\subsection{Propagation mode}

The main route of transmission of COVID-19 is through droplets or aerosols produced when coughing and sneezing. In the early stages of infection, the high expression of ACE2 in the mouth and tongue promotes the invasion of SARS-CoV-2[15].

New research revealed that temperature and relative humidity were both negatively related to daily new cases and deaths. A $1{ }^{\circ} \mathrm{C}$ increase in temperature was associated with a $3.08 \%(95 \% \mathrm{CI}: 1.53 \%, 4.63 \%)$ reduction in daily new cases and a $1.19 \%(95 \% \mathrm{CI}: 0.44 \%, 1.95 \%)$ reduction in daily new deaths, whereas a $1 \%$ increase in relative humidity was associated with a $0.85 \%$ (95\% CI: $0.51 \%$, $1.19 \%)$ reduction in daily new cases and a $0.51 \%(95 \%$ CI: $0.34 \%, 0.67 \%$ ) reduction in daily new deaths. These findings provide that the COVID-19 pandemic may be partially suppressed with temperature and humidity increases [16].

\section{Non-drug intervention measures}

COVID-19 is a newly emerging infectious disease. In the early stage of the outbreak, there was a lack of knowledge about the infectivity, pathogenicity, and virulence of novel coronavirus. There was a lack of effective therapeutic drugs and vaccines that could be used for preventive inoculation. Early outbreak COVID - 19 pandemic control main non-drug intervention measures, including patients and asymptomatic infection monitoring report, the isolation treatment, cure health after discharge follow-up and monitoring, close contact tracing, testing and medical observation, key population screening high-risk, foci disinfection, wearing a mask, wash your hands often and keep personal protective measures such as social distance [17]. In the face of the epidemic, specific nonpharmaceutical interventions are as follows:

\subsection{Case detection}

Mass testing to detect and isolate asymptomatic cases is an important strategy to contain the spread of the virus. Nucleic acid tests and antibody tests are the most commonly used methods for case detection. Nucleic acid testing is currently the gold standard for detecting COVID-19 patients, but its accuracy is affected by various external factors, which may lead to a missed diagnosis of COVID-19. The combination of nucleic acid testing and antibody testing can improve the positive detection rate of COVID-19 [18].

\subsection{Case isolation}

Case isolation refers to the separation of those diagnosed with infectious diseases from those who do not. Isolation in the early stage of the epidemic can control the influence of individual activities on transmission. The premise of successful isolation is timely and comprehensive contact tracing of each confirmed patient. The quarantine can be done at home or in a designated place [18]. Today, timely case detection and case isolation remain the main public health interventions.

\subsection{Isolation of close contacts}

Close contact isolation is the activity of isolating and restricting contacts of possible cases for medical observation to determine if they are infected with the virus, thereby reducing their risk of infecting others. Specific measures include movement restrictions, ideally combined with medical observation. The effectiveness of the measures depends on the feasibility of isolating contacts regarding the sequence interval (the time interval between the onset of clinical symptoms in the first generation of cases and the onset of clinical symptoms in the second generation of cases), the number of infected 
persons. According to a rapid review by Cochrane [19], 10 studies that simulated COVID-19 in China, the United Kingdom, Korea, and on the Diamond Princess cruise ship all found that this measure successfully reduced the morbidity and mortality during the COVID-19 pandemic, reducing the number of cases by $44 \%$ to $81 \%$ and the number of deaths by $31 \%$ to $63 \%$. At the same time, it reduces the cost of epidemic prevention. At the same time, individuals should be kept in isolation for as long as possible as required, and close contacts should be provided with clear reasons for their isolation and sufficient supplies for their use during the isolation period [18].

\subsection{Close contact tracing}

According to the World Health Organization (WHO) [20], contact tracing refers to monitoring and tracing close contacts of confirmed cases to prevent further transmission of the virus. The outbreak has been tracked both digitally (based on mobile apps) and manually (based on phone interviews). This measure is essential to reduce the risk of widespread transmission within the community. An optimized contact tracking strategy can greatly reduce the number of infections. However, data tracking has privacy violations and needs to be understood and supported by the public before it can be better implemented [18].

\subsection{Wear a mask}

Wearing masks significantly reduced the amount of influenza virus RNA in respiratory droplets and coronavirus RNA in aerosols. This measure reduces the risk of novel coronavirus infection in the population and reduces the transmission of other respiratory viruses [18].

\subsection{Expand social distance}

Keeping away from the gathering places of crowds and keep a distance of at least 2 meters from others. One is to avoid crowded places (suspension of classes and work, restriction of gathering, traffic control, closing at home). The other is to keep a safe distance from people when forced to go out [18].

\subsection{Travel restrictions}

Travel restrictions include border closures, entry and exit restrictions, and traffic restrictions. This is good for preventing infected people from spreading the virus further afield but bad for the tourist economy [18].

These are the details of non-drug interventions, which largely prevented the spread of the epidemic and reduced the spread of novel coronavirus. However, due to the different understanding of the epidemic in different countries, different countries' prevention and control mode is also different. In terms of the severity and looseness of epidemic prevention and control, the epidemic prevention and control models of countries around the world can be divided into three categories:
China's national tightly targeted prevention and control model; Europe's resilient control model; America's liberal-loose control model.

\section{DIAGNOSES}

\subsection{Nucleic acid assay}

\subsubsection{Whole genome sequencing}

At present, the most widely used gene sequencing technology is high-throughput sequencing (HTS), also called next-generation sequencing (NGS) [21]. Clinical metagenomic next-generation sequencing (mNGS) comprehensively analyzes microbial and host genetic material (DNA or RNA) in samples from patients. It allows for the identification and genomic characterization of bacteria, fungi, parasites, and viruses without the need for prior knowledge of a specific pathogen directly from clinical specimens [22]. The technique involves first preparing viral RNA into a DNA library that can be analyzed by sequencers and simultaneously detecting large nucleic acid sequences using sequencers [21]. This mechanic has made a great contribution to the identification of a new virus. Within 2 to 3 weeks after the outbreak in Wuhan, Chinese scientists identified a novel coronavirus by gene sequencing method and successfully mapped its entire genome sequence information [21]. However, due to the complicated operation, relatively long detection time $(24 \sim 72 \mathrm{~h})$, and relatively low detection sensitivity of MNGS, it is not easy to achieve large-scale promotion and rapid diagnosis to detect novel coronavirus [23].

\subsubsection{RT-qPCR}

Real-time Quantitative polymerase chain reaction first converts the extracted viral genomic RNA into complementary DNA (cDNA) through reverse transcription. It then uses pathogen-specific primers to amplify the nucleic acid sequence of the pathogen using cDNA as a template. During the amplification process, fluorescent dyes can synchronously integrate into the product. In the exponential period of PCR amplification, there is a linear relationship between the CT value of the template and the initial copy number of the template, so it becomes the basis of quantitative analysis. RT-PCR is minimally invasive because it can perform using nasal swabs, throat swabs, and saliva or other bodily fluids tests. The experiment also allows for social distancing: while some molecular tests, including RT-PCR, are sometimes conducted in a hospital or clinic, swabs can also be taken from the patient's home [24]. Moreover, RT-PCR diagnosis takes less time and has more samples. Moreover, the result is simple and rapid, and the detection cost is low, which is especially suitable for the COVID-19 epidemic. Improvements to this detection method are numerous. The multiple fluorescent RT-PCR detection kit independently developed by Prof. Ying Bin Wu and Prof. Ke Bo Wen, and Prof. Geng Jia from West China Hospital collaborated 
with the conserved sequences of novel coronavirus ORFIab gene and $\mathrm{N}$ gene as targets, designed specific primers and oligonucleotide fluorescent probes to detect viral nucleic acid with high specificity [25]. In Hong Kong university of science and technology, a team led by professor Wen Wei Jia developed a new type of silicon micro heater module; it has a lower thermal quality and a good thermal conductivity. Temperature speeds up from traditional PCR device in average $4-5{ }^{\circ} \mathrm{C}$ to $30{ }^{\circ} \mathrm{C}$ per second, to shorten the testing time [26]. Researchers at the Hong Kong Polytechnic University have developed a diagnostic system that can detect up to 40 infectious respiratory pathogens in one hour with a single test [27]. But the improvements made to reduce the time consumption cannot compensate for the complexity of RT-PCR. Nucleic acid testing is a complex procedure that can take hours. Moreover, the test results need to be reviewed. It takes more time to repeat the experiment, which causes many patients not being isolated and treated in time before the diagnosis is made. In addition, due to low viral content in oropharyngeal swabs and the easy degradation of viral RNA, nucleic acid tests often show false-negative results.

\subsection{Serological tests}

\subsubsection{Enzyme-linked immunosorbent assay}

By testing positive for novel coronavirus-specific $\operatorname{IgM}$ antibodies and $\mathrm{IgG}$ antibodies, the laboratory can confirm whether a sample corresponds to an individual carrying the virus. Enzyme-linked immunosorbent assay (ELISA) is a kind of immunological assay characterized by intense specificity, high sensitivity, and stable results [28]. It combines the soluble antigen or antibody to the solid phase carrier such as polystyrene and uses the specific antigen and antibody to carry out the immune reaction. $\operatorname{IgM}$ is the first antibody to appear in the primary humoral immune response. Elevated IgM in the serum indicates a recent infection. $\mathrm{IgG}$ is the antibody with the highest serum and body fluids content, accounting for $75-80 \%$ of the total serum Ig. IgG is the main antibody produced by the re-immune response, with high affinity and wide distribution in the body, and has significant immune effects. The incubation period of the novel coronavirus infection is 0-14 days, and the immune system of the body will produce antivirus-specific IgM and IgG antibodies one after another. Detection of the presence of specific antibodies in the patient's serum can serve as a diagnostic index for novel coronavirus infection. It is generally believed that specific IgM can be found in the patient's serum 7 days after infection and IgG 10 days after infection. To conduct one ELISA test, the $\mathrm{N}$ protein of the novel coronavirus gene was amplified and purified to be ready for use. After the coating, washing, sealing, and washing of the $\mathrm{N}$ protein solution, the serum to be tested was added to conduct the antigen-antibody reaction. The substrate was added to determine the absorbance value after color development. A novel coronavirus positive is diagnosed when the absorbance value is above the set threshold. The advantages of ELIZA are that clinical specimens are easy to collect, which greatly reduces the risk of getting an infection when collecting samples. The test has high specificity and sensitivity. Additionally, the instrument is easy to operate and has been obtained in various hospitals. Continued monitoring of $\operatorname{IgM} / \operatorname{IgG}$ after treatment can provide important prognostic indicators for physicians. Its disadvantage is that the patient is infected 7-14 days later, will appear in the serum IgM, IgG antibody, still can't achieve the purpose of early diagnosis.

\subsection{Other methods}

\subsubsection{CT scanning}

Researchers from Shanghai Jiao Tong University noted that both COVID-19 and influenza virus pneumonia are caused by respiratory viruses and have similar symptoms and signs. That makes it difficult to distinguish the two diseases based on symptoms and routine blood tests; however, the implementation of CT scanning can further determine the symptoms [29]. Insufficient nucleic acid testing and high false-negative rates prompt clinicians to enhance the role of CT imaging for accurate analysis in differential diagnosis and radiological artificial intelligence [30].

Generally speaking, nucleic acid assay and serological tests are the main streams of diagnosis. With the help of CT scanning and other mechanics, an infected case can be further certainly determined. However, though every method has strengths and apparent benefits, each of them contains weaknesses as well. Researches have been introduced to amend and build up better testing methods. Furthermore, other methods are also used, and new, more efficient strategies are always required.

\section{Treatments}

Treatments for COVID-19 include drugs, vaccines, and mechanical systems. In this section, deferoxamine mesylate using for repressing immune response, the difference between mRNA vaccine and inactivated vaccine, and life-supporting system to encounter complications are specially discussed.

\subsection{Drug: Iron Chelator (Desferal)}

Various drugs provide a comparably simple and affordable way to treat COVID-19. In the following section, desferal is discussed from three perspectives-safety, mechanism, and affordability.

\subsubsection{Clinical Trial}

According to the U.S. National Library of Medicines [31], this drug is on phase 2 of a clinical trial, sponsored by Kermanshah University of Medical Sciences since April 2020. According to the regulation of clinical trials, desferal treatment has passed the first two trials for safety and efficiency. Dr. Yadollah Shakiba, the experiment director [31], stated that "COVID-19 with mild, moderate 
or severe pneumonia will be treated with standard treatment regimens in combination with IV injection of Deferoxamine".

\subsubsection{Mechanism}

Iron Chelator is a drug that reduces the iron level in organisms, which helps deal with viral infections. According to Duchemin J. and Paradkar P. (2020) [31], mosquitoes treated with Iron Chelator show fewer vital titers because the drug significantly reduces intracellular iron levels. For mosquitoes, Duchemin J. and Paradkar P. (2020) stated that "heavy chain ferritin may be part of an immune mechanism of mosquitoes in response to viral infections".

\subsubsection{Affordability}

The price reported by MedChemExpress [32] induced that desferal was an affordable drug for the most since $100 \mathrm{mg}$ Iron Chelator only caused $¥ 500$.

\subsection{Vaccines}

To be fully prepared for the next peak of COVID-19 infection, scientists worldwide are struggling to invent vaccines to treat it. There are various types of vaccines being experimented on. In the following section, mRNA vaccines and inactivated vaccines and their advantages are specifically discussed [33].

\subsection{Treatments for complications}

Complications of COVID-19 are largely different in severity, scale, and probability. The most serious one that takes most patients' life is dyspnea. Other than drugs and vaccines, life-supporting systems are specifically prepared for patients who suffer severe COVID-19 infection. In the following section, extra-corporeal membrane oxygenation, shorten for ECMO and endotracheal tube operation, and their mechanisms are discussed.

\subsubsection{Life-supporting system: ECMO}

\subsubsection{Mechanism}

Extracorporeal membrane oxygenation, ECMO, provides life support for patients having difficulty breathing or having heart failure. Dyspnea and heart failure can lead to a severe drop in blood oxygen saturation, which can eventually lead to death. ECMO.

\subsubsection{Application}

On 22nd January, the Hospital of Wuhan University [34] successfully saved a patient infected by COVID-19 by using ECMO. Ever since then, tens of ECMOs have been sent to Wuhan for COVID-19 treatment. The concern on ECMO training can be seen elsewhere in the world.
According to Brei. B., an experiment was conducted to determine if the ECMO training providers had successfully spread the knowledge of using an ECMO when needed.

\subsubsection{Endotracheal tube}

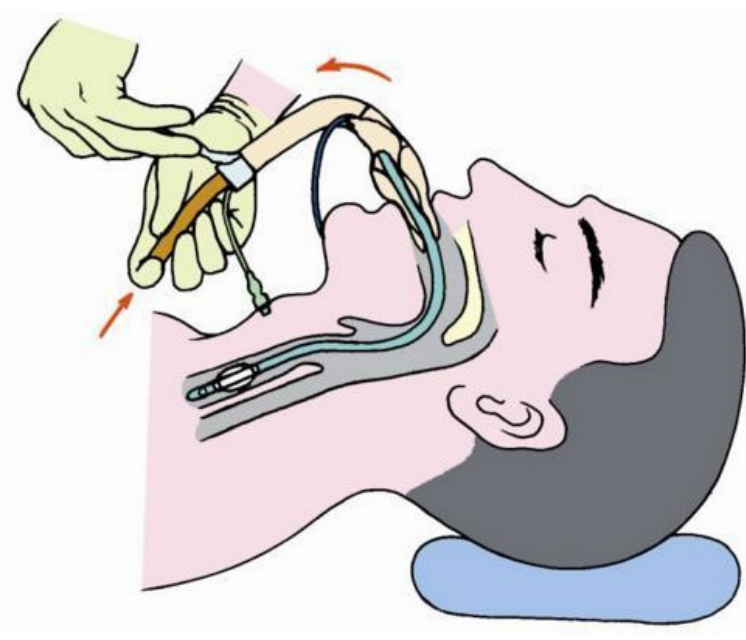

Endotracheal tube

\subsubsection{Tips for safe and successful endotracheal tube operation}

Gently move through the throat to avoid extra destruction on teeth and edema of the larynx. Check if the patient has loose teeth before the operation. If there is any hard object that can fall during the operation, operators should remove them. Normally an X-ray exam should be conducted to check if the endotracheal tube is at the right position [35].

\section{Conclusion}

We have presented the genome, and pathogenic information of novel coronavirus elaborated certain actions to prevent the transmission of the infectious disease, and introduced ways to detect the occurrence of the virus. It is known that the genome contains 29,903 bases, while the $\mathrm{S}$ protein it coded is a multifunctional molecular machine that mediates coronavirus entry into host cells. The invasion of COVID19 is mainly through the receptor-binding domain on the virus and the related receptors on the target cell, using angiotensin-converting ACE2 on the cell surface as the corresponding recognition receptor. Early outbreak preventions include patients and asymptomatic infection monitoring report, the isolation treatment, cure health after discharge follow-up and monitoring, close contact tracing, testing and medical observation, key population screening high-risk, foci disinfection, wearing a mask, wash your hands often, and keep personal protective measures. The virus tests mainly implemented these days consist of nucleic acid assay and serological testings with the assistance of other proper methods. The epidemic shows little signs to end so far. 
Thus, future investigations of the effective treatment of this intractable illness are always expected by the general public.

\section{References}

1. Wu JT, Leung $\mathrm{K}$, Leung GM. Nowcasting and forecasting the potential domestic and international spread of the 2019-nCoV outbreak originating in Wuhan, China: a modelling study. Lancet, 2020, 395(10225): 689-697

2. Kim GU, Kim MJ, Ra SH, Lee J, Bae S, Jung J, et al. Clinical characteristics of asymptomatic and symptomatic patients with mild COVID-19. Clin Microbiol Infect. 2020; S1198-743X (20) 30268-8.

3. Lu R, Zhao X, Li J, et al. Genomic characterisation and epidemiology of 2019 novel coronavirus: implications for virus origins and receptor binding. Lancet, 2020, 395(10224): 565-574.

4. Chen Y, Liu Q, Guo D. Emerging coronaviruses: genome structure, replication, and pathogenesis. J Med Virol, 2020, 92(4): 418-423.

5. Zhou P, Yang XL, Wang XG, et al. A pneumonia outbreak associated with a new coronavirus of probable bat origin. Nature, 2020, 579(7798): 270273.

6. Chan JF, Kok KH, Zhu Z, et al. Genomic characterization of the 2019 novel human-pathogenic coronavirus isolated from a patient with atypical pneumonia after visiting Wuhan. Emerg Microbes Infect, 2020, 9(1): 221-236.

7. Perlman S, Netland J. Coronaviruses post-SARS: update on replication and pathogenesis. Nat Rev Microbiol, 2009, 7(6): 439-450.

8. Li F. Structure, function, and evolution of coronavirus spike proteins. Annu Rev Virol, 2016, 3(1): 237-261.

9. Hoffmann M, Kleine-Weber H, Schroeder S, et al. SARS-CoV-2 cell entry depends on ACE2 and TMPRSS2 and is blocked by a clinically proven protease inhibitor. Cell, 2020, 181(2): 271-280, e278.

10. Walls AC, Park YJ, Tortorici MA, et al. Structure, function, and antigenicity of the SARS-CoV-2 spike glycoprotein. Cell, 2020, 183(6): 1735.

11. Wrapp D, Wang N, Corbett KS, et al. Cryo-EM structure of the 2019-nCoV spike in the prefusion conformation. Science, 2020, 367(6483): 1260-1263.

12. Li Q, Guan $\mathrm{X}, \mathrm{Wu} \mathrm{P}$, et al. Early transmission dynamics in Wuhan, China, of novel coronavirusinfected pneumonia. N Engl J Med, 2020, 382(13): 1199-1207.

13. Hou YJ, Okuda K, Edwards CE, et al. SARS-CoV-2 reverse genetics reveals a variable infection gradient in the respiratory tract. Cell, 2020, 182(2): 429-446, e414.

14. Xu Z, Shi L, Wang Y, et al. Pathological findings of COVID-19 associated with acute respiratory distress syndrome. Lancet Respir Med, 2020, 8(4): 420-422.
15. Xu H, Zhong L, Deng J, et al. High expression of ACE2 receptor of 2019-nCoV on the epithelial cells of oral mucosa. Int J Oral Sci, 2020, 12(1): 8.

16. Wu Y, Jing W, Liu J, et al. Effects of temperature and humidity on the daily new cases and new deaths of COVID-19 in 166 countries. Sci Total Environ. 2020 Aug 10;729:139051. doi: 10.1016/j.scitotenv.2020.139051. Epub 2020 Apr 28. PMID: 32361460; PMCID: PMC7187824.

17. Liu Wenge, Yang Zhiwen.Global public health governance dilemma and response strategy under the COVID-19 [J]. Regional and global development,2020,4(05):68-80+156-157.

18. Li Cheng, Zhong Yang, Wu Yi, Yang Changhong, Zhou Lijun, Zhang Tao.Current situation and prospect of non-drug intervention for COVID-19 epidemic [J]. Modern Preventive Medicine, 201,48(03):385-388.

19. Nussbaumer - Streit B, Mayr V, Dobrescu A, et al. Quarantine alone or in combination with other public health measures to control COVID - 19: a rapid review [J]. The Cochrane Database of Systematic Reviews, 2020, 4(4): CD013574.

20. World Health Organization. Contact tracing [ EB/OL ] . [ [ 2021 - $01-03]$. https:/www.who.int/csr/resources/publications/ebol a/contact-tracing-guidelines/en/.

21. Wang J, Gao SY, Zhu RX, et al. Main detection methods of SARS-CoV-2 [J]. Chin J Comp Med, 2021, 31(1): 120-124. doi: 10. 3969/j. issn. 16717856. 2021. 01.018

22. Chiu, Charles Y.; Miller, Steven A. (June 2019). "Clinical metagenomics". Nature Reviews Genetics. 20 (6): 341-355. doi:10.1038/s41576-019-0113-7

23. Tao Yue, Fu Qihua, Mo Qian. The application of metagenomic sequencing in the detection of novel coronavirus [J]. Chin J Lab Med, 2020, 43(3): 217220.

24. Brandon May. Pros and Cons of the Common Types of COVID-19 Tests [EB/OL]. https://www.biospace.com/article/pros-and-cons-ofthe-common-types-of-covid-19-tests/, Oct 15, 2020.

25. Ke Bo Wen. The joint research team of science and technology in West China Hospital has successfully developed several novel Coronavirus detection kits [R/OL]. West China Hospital of Sichuan University, (2020-02-19)

[2020-04-25]. http://www.wchscu.cn/comprehensive/50848.html

26. HKKUST. HKUST Research Team Invents World's Fastest Coronavirus Detection Device Offering Diagnostic Results in 40 Minutes. [2020-02-07].

27. Terence Lau Lokting. PolyU develops the world 's most comprehensive rapid, automated multiplex diagnostic system for detecting up to 40 infectious respiratory pathogens (including novel coronavirus) in a single test. [2020-02-11]. 
28. Wu T, Ma J. Novel coronavirus immunoassay -ELISA method [EB/OL]. Chinese Society of Immunology, 2020-02-14.

29. Zhou M,Chen Y,Yang D X, et al. A Deep Learning Pipeline for Accurate Differential Diagnosis between Novel Coronavirus Pneumonia and Influenza Pneumonia. Preprints with The Lancet. [2020-02-19].

30. Xie X Z, Zhong Z,Zhao W, et al. Chest CT for Typical 2019- nCoV Pneumonia: Relationship to Negative RT-PCR Testing Radiology. [2020-02-12].

31. Shakiba Y. (2020). U.S. National Library of Medicine. Application of Desferal to treat COVID-19. https://www.clinicaltrials.gov/ct2/show/NCT043335 50.

32. MedChemExpress. Deferoxamine mesylate. https://www.medchemexpress.cn/Deferoxaminemesylate.html.

33. Callaway E. (2020). The Race for Coronavirus Vaccines: a Graphical Guide. Nature. https://www.nature.com/articles/d41586-020-01221y.

34. Brei B. et.al. (2020) Inter-professional ECMO Telerounding: a Novel Approach to Neonatal ECMO Clinical Participation and Education. Nature. https://www.nature.com/articles/s41372-020-008274.

35. Baike. Sogou. Endotracheal Tube. https://baike.sogou.com/v6386903.htm. 